\title{
ASYMPTOTIC ALMOST AUTOMORPHY OF FUNCTIONS AND DISTRIBUTIONS
}

\author{
Chikh Bouzar \\ Laboratory of Mathematical Analysis and Applications, University of Oran 1, \\ Ahmed Ben Bella, 31000, Oran, Algeria \\ ch.bouzar@gmail.com \\ Fatima Zahra Tchouar \\ University Center of Ain Temouchent, 46000 Ain Temouchent, Algeria \\ fatima.tchouar@gmail.com

\begin{abstract}
This work aims to introduce and to study asymptotic almost automorphy in the context of Sobolev-Schwartz distributions. Applications to linear ordinary differential equation and neutral difference differential equations are also given.

Keywords: Asymptotically almost automorphic functions, Asymptotically almost automorphic distributions, Neutral difference differential equations.
\end{abstract}

\section{Introduction}

The paper aims to study asymptotic almost automorphy in the context of functions and Sobolev-Schwartz distributions, it is well known that the concept of almost automorphy is strictly more general than the almost periodicity studied in a full generality by H. Bohr, see [4] and [8]. The concept of asymptotic almost periodicity as a perturbation of almost periodic functions by functions that vanish at infinity belongs to M. Fréchet in [9], one of the main motives of which is the introduction of this concept in obtaining the existence of an almost periodic solution to differential equations if they admit an asymptotic almost periodic solution. In the same vein as Fréchet motivation, we study the existence of solutions of linear neutral difference differential equations with variable coefficients in the framework of asymptotically almost automorphic distributions. Almost periodicity in the framework of distributions extending the classical Bohr and Stepanov almost periodicity [16] is considered by L. Schwartz [13]. The paper [7] deals with asymptotic almost periodicity of distributions.

In [1] and [3], S. Bochner defined explicitly almost automorphic functions, where some basic properties have been established. He studied linear difference differential equations in the framework of almost automorphic functions in [2]. Almost automorphy of primitives and asymptotically almost automorphic functions are also considered, see $[12,18]$.

We first investigated the almost automorphy in the settings of distributions and generalized functions respectively in [6] and [5], then we addressed the issue of asymptotic almost automorphy in these contexts, see the communication [17].

The paper is organized as follows: the second section studies asymptotically almost automorphic functions following an appropriate definition, essential properties of these functions are proved; the third section deals with smooth asymptotically almost automorphic functions. The fourth section is dedicated to asymptotically almost automorphic distributions; we give their definition, 
characterizations and some of their properties. The last section is an application to linear neutral difference differential equations of asymptotically almost automorphic distributions.

\section{Asymptotically almost automorphic functions}

It is worth noting that the definition of an asymptotically almost automorphic function depends on the choice of authors, but in general the essential idea of the decomposition in the definition of an asymptotically almost automorphic function is preserved. The differences in their definitions lie in the domain of definition of the considered functions, their regularity and finally in the choice of the interval of decomposition. We consider functions defined, continuous and bounded on the whole space of real numbers $\mathbb{R}$ and the decomposition on the closed interval $[0,+\infty[$. So, we have to precise some results on asymptotically almost automorphic functions. Let $\mathcal{C}_{b}$ denotes the space of bounded and continuous complex-valued functions defined on $\mathbb{R}$, endowed with the norm $\|\cdot\|_{\infty}$ of uniform convergence on $\mathbb{R}$, it is well-known that $\left(\mathcal{C}_{b},\|\cdot\|_{\infty}\right)$ is a Banach algebra. Let $\omega \in \mathbb{R}$ and $f, \varphi$ functions, we recall that the translation operator $\tau_{\omega}$ is defined by $\tau_{\omega} f(\cdot)=f(\cdot+\omega)$, and $\check{\varphi}$ by $\check{\varphi}(x)=\varphi(-x)$. Denote $\mathbb{J}:=[0,+\infty[$.

Definition 1. The space $\mathcal{C}_{+, 0}$ is the set of all bounded and continuous complex-valued functions defined on $\mathbb{R}$ and vanishing at $+\infty$.

We give some properties of the space $\mathcal{C}_{+, 0}$ which are proved in a straight way.

Proposition 1. The following is true:

(1) The space $\mathcal{C}_{+, 0}$ is a Banach subalgebra of $\mathcal{C}_{b}$.

(2) $\tau_{\omega} \mathcal{C}_{+, 0} \subset \mathcal{C}_{+, 0}, \forall \omega \in \mathbb{R}$.

(3) $\mathcal{C}_{+, 0} \times \mathcal{C}_{b} \subset \mathcal{C}_{+, 0}$

(4) $\mathcal{C}_{+, 0} * L^{1} \subset \mathcal{C}_{+, 0}$

(5) Let $h \in \mathcal{C}_{+, 0}$, if $h^{\prime}$ exists and is uniformly continuous on $\mathbb{J}$, then there exists a function $H \in \mathcal{C}_{+, 0}$ such that $H=h^{\prime}$ on $\mathbb{J}$.

(6) There exists $H \in \mathcal{C}_{+, 0}$ a primitive of $h$ on $\mathbb{J}$ if and only if $\int_{0}^{+\infty} h(t) d t<\infty$ and $\int_{0}^{x} h(t) d t$ is bounded on $\mathbb{J}$.

Remark 1. In (5) if $h^{\prime}$ exists and is uniformly continuous on $\mathbb{R}$, then $H=h^{\prime}$ on $\mathbb{R}$.

Remark 2. If $h$ is a locally integrable function, we denote by $\int_{0}^{+\infty} h(t) d t$ the improper integral, and $\int_{0}^{+\infty} h(t) d t<\infty$ means $\int_{0}^{+\infty} h(t) d t$ is finite.

Recall some properties of almost automorphic functions, see $[1,3,12,18]$.

Definition 2. A complex-valued function $g$ defined and continuous on $\mathbb{R}$ is called almost automorphic if for any sequence $\left(s_{m}\right)_{m \in \mathbb{N}} \subset \mathbb{R}$, one can extract a subsequence $\left(s_{m_{k}}\right)_{k}$ such that

$$
\tilde{g}(x):=\lim _{k \rightarrow+\infty} g\left(x+s_{m_{k}}\right) \quad \text { exists for every } \quad x \in \mathbb{R},
$$


and

$$
\lim _{k \rightarrow+\infty} \tilde{g}\left(x-s_{m_{k}}\right)=g(x) \quad \text { for every } \quad x \in \mathbb{R} .
$$

The space of almost automorphic functions on $\mathbb{R}$ is denoted by $\mathcal{C}_{a a}$.

Remark 3. The function $\tilde{g}$ is not necessary continuous but $\tilde{g} \in L^{\infty}(\mathbb{R})$.

Proposition 2. The following is true:

(1) The space $\mathcal{C}_{a a}$ is a Banach subalgebra of $\mathcal{C}_{b}$.

(2) $\tau_{\omega} \mathcal{C}_{a a} \subset \mathcal{C}_{a a}, \forall \omega \in \mathbb{R}$

(3) $\mathcal{C}_{a a} * L^{1} \subset \mathcal{C}_{a a}$.

(4) $\mathcal{C}_{a a} \cap \mathcal{C}_{+, 0}=\{0\}$

(5) A primitive of an almost automorphic function is almost automorphic if and only if it is bounded.

We give now the definition of an asymptotically almost automorphic function.

Definition 3. We say that a function $f \in \mathcal{C}_{b}$ is asymptotically almost automorphic, if there exist $g \in \mathcal{C}_{a a}$ and $h \in \mathcal{C}_{+, 0}$ such that $f=g+h$ on $\mathbb{J}$. The space of asymptotically almost automorphic functions is denoted by $\mathcal{C}_{\text {aaa }}$.

Example 1. $\mathcal{C}_{a a} \subset \mathcal{C}_{a a a}$ and $\mathcal{C}_{+, 0} \subset \mathcal{C}_{a a a}$.

It can be seen easly that the decomposition of an asymptotically almost automorphic function is unique on $\mathbb{J}$, so if $f \in \mathcal{C}_{a a a}$ and $f=g+h$ on $\mathbb{J}$, where $g \in \mathcal{C}_{a a}$ and $h \in \mathcal{C}_{+, 0}$, the function $g$ is said the principal term of $f$ and the function $h$ is the corrective term of $f$, we denote them respectively by $f_{a a}$ and $f_{c o r}$. Then the notation $f=\left(f_{a a}+f_{c o r}\right) \in \mathcal{C}_{a a a}$ means that $f_{a a} \in \mathcal{C}_{a a}, f_{c o r} \in \mathcal{C}_{+, 0}$ and $f=f_{a a}+f_{c o r}$ on $\mathbb{J}$.

Proposition 3. The following is true:

(1) $\tau_{\omega} \mathcal{C}_{a a a} \subset \mathcal{C}_{a a a}, \forall \omega \in \mathbb{R}_{+}$

(2) $\mathcal{C}_{a a a} \times \mathcal{C}_{a a} \subset \mathcal{C}_{a a a}$

(3) $\mathcal{C}_{a a a} * L^{1} \subset \mathcal{C}_{a a a}$.

(4) Let $f \in \mathcal{C}_{a a a}$ and $\phi$ is a continuous function on $\mathbb{C}$, then $\phi \circ f \in \mathcal{C}_{a a a}$.

(5) If $f=\left(f_{a a}+f_{\text {cor }}\right) \in \mathcal{C}_{a a a}$, then $\left\|f_{a a}\right\|_{\infty} \leq \sup _{x \in \mathbb{J}}|f(x)|$. In particular, for $f \in \mathcal{C}_{a a}$ and $\omega \in \mathbb{R},\|f\|_{\infty}=\sup _{x \geq \omega}|f(x)|$.

(6) Let $\left(f_{m}\right)_{m \in \mathbb{N}}=\left(f_{m, a a}+f_{m, c o r}\right)_{m} \subset \mathcal{C}_{a a a}$ converges uniformly on $\mathbb{J}$ to a function $f$, then there exists $\phi=(g+h) \in \mathcal{C}_{a a a}$, such that $\phi=f$ on $\mathbb{J}, g \in \mathcal{C}_{a a}$ is the uniform limit on $\mathbb{R}$ of $\left(f_{m, a a}\right)_{m}$ and $h \in \mathcal{C}_{+, 0}$ is the uniform limit on $\mathbb{I}$ of $\left(f_{m, c o r}\right)_{m}$. 
P r o o f. The proofs of (1) and (2) are easy.

(3) Let $\psi \in L^{1}$ and $f=\left(f_{a a}+f_{c o r}\right) \in \mathcal{C}_{a a a}$. Since $f=f_{a a}+\left(f-f_{a a}\right)$, where $\left(f-f_{a a}\right) \in \mathcal{C}_{+, 0}$, it follows from Proposition 2-(3) and Proposition 1-(4) that $f * \psi \in \mathcal{C}_{a a a}$. Now we show explicitly the principal part and the corrective part of $f * \psi$. For $x \in \mathbb{J}$, we have

$$
\begin{aligned}
(f * \psi)(x)= & \int_{\mathbb{R}} f(y) \psi(x-y) d y=\int_{-\infty}^{0} f(y) \psi(x-y) d y+\int_{0}^{+\infty}\left(f_{a a}(y)+f_{c o r}(y)\right) \psi(x-y) d y \\
& =\left(f_{a a} * \psi\right)(x)+\left(f_{c o r} * \psi\right)(x)+\int_{-\infty}^{0}\left(f-f_{a a}-f_{c o r}\right)(y) \psi(x-y) d y
\end{aligned}
$$

By Proposition 2-(3), $\left(f_{a a} * \psi\right) \in \mathcal{C}_{a a}$ and by Proposition $1-(4),\left(f_{c o r} * \psi\right) \in \mathcal{C}_{+, 0}$. On the other hand, for $x \in \mathbb{R}$,

$$
\int_{-\infty}^{0}\left(f-f_{a a}-f_{c o r}\right)(y) \psi(x-y) d y=\int_{\mathbb{R}}\left(f-f_{a a}-f_{c o r}\right)(x-y) \chi_{] x,+\infty[}(y) \psi(y) d y .
$$

It is easy to see that the latter function is continuous and bounded on $\mathbb{R}$ and by the dominated convergence theorem it vanishes at infinity. Then $f * \psi=\left(\Psi_{a a}+\Psi_{c o r}\right) \in \mathcal{C}_{a a a}$, where $\Psi_{a a}:=f_{a a} * \psi$ and $\Psi_{c o r}:=f_{c o r} * \psi+\int_{-\infty}^{0}\left(f-f_{a a}-f_{c o r}\right)(y) \psi(.-y) d y$.

(4) Let $f=\left(f_{a a}+f_{c o r}\right) \in \mathcal{C}_{a a a}$ and $\phi$ be a continuous function on $\mathbb{C}$, then it is well-known that $\phi(f) \in \mathcal{C}_{b}$ and also $\phi\left(f_{a a}\right) \in \mathcal{C}_{a a}$. On the other hand, it is easy to see that the function $\phi(f)-\phi\left(f_{a a}\right)$ defined on $\mathbb{R}$ belongs to $\mathcal{C}_{+, 0}$. Consequently we have $\phi(f)=\left(\phi(f)_{a a}+\phi(f)_{c o r}\right) \in \mathcal{C}_{a a a}$, where $\phi(f)_{a a}=\phi\left(f_{a a}\right)$ and $\phi(f)_{c o r}=\phi(f)-\phi\left(f_{a a}\right)$.

(5) Let $f=\left(f_{a a}+f_{\text {cor }}\right) \in \mathcal{C}_{a a a}$ and $\left(s_{m_{k}}\right)_{k}$ a subsequence of $\left(s_{m}\right)_{m \in \mathbb{N}} \subset \mathbb{J}$ which tends to infinity. Let $x \in \mathbb{R}$ and $k_{0} \in \mathbb{Z}_{+}$such that the sequence $\left(x+s_{m_{k}}\right)_{k \geq k_{0}} \subset \mathbb{J}$ tends to infinity, then for $k \geq k_{0}$, we have

$$
\left|f_{a a}\left(x+s_{m_{k}}\right)\right| \leq\left|f\left(x+s_{m_{k}}\right)\right|+\left|f_{\text {cor }}\left(x+s_{m_{k}}\right)\right| \leq \sup _{x \in \mathbb{J}}|f(x)|+\left|f_{c o r}\left(x+s_{m_{k}}\right)\right|
$$

so $\forall x \in \mathbb{R}$,

$$
\left|\tilde{f}_{a a}(x)\right|=\lim _{k \rightarrow+\infty}\left|f_{a a}\left(x+s_{m_{k}}\right)\right| \leq \sup _{x \in \mathbb{J}}|f(x)|
$$

It follows then

$$
\left|f_{a a}(x)\right|=\lim _{k \rightarrow+\infty}\left|\tilde{f}_{a a}\left(x-s_{m_{k}}\right)\right| \leq \sup _{x \in \mathbb{J}}|f(x)|, \quad \forall x \in \mathbb{R} .
$$

Consequently, we obtain the results.

(6) Let $\left(f_{m}\right)_{m}=\left(f_{m, a a}+f_{m, c o r}\right)_{m} \subset \mathcal{C}_{a a a}$ converges uniformly to $f$ on $\mathbb{J}$, by (5) we have

$$
\left\|f_{n, a a}-f_{m, a a}\right\|_{\infty} \leq \sup _{x \in \mathbb{J}}\left|f_{n}(x)-f_{m}(x)\right|,
$$

hence $\left(f_{m, a a}\right)_{m \in \mathbb{N}}$ is a Cauchy sequence in the Banach space $\mathcal{C}_{a a}$, i.e. $\left(f_{m, a a}\right)$ converges uniformly on $\mathbb{R}$ to a function $g \in \mathcal{C}_{a a}$. Let's define the function $h$ by

$$
h(x)= \begin{cases}(f-g)(x), & x \geq 0 \\ (f-g)(0), & x<0\end{cases}
$$


Then $h \in \mathcal{C}_{b}$ and $\left(f_{m, c o r}\right)_{m}$ converges uniformly on $\mathbb{J}$ to $h$, i.e. $\lim _{x \rightarrow+\infty} h(x)=0$ hence $h \in \mathcal{C}_{+, 0}$. Define $\phi=g+h$ on $\mathbb{R}$, then $\phi \in \mathcal{C}_{a a a}$ and $\phi=f$ on $\mathbb{J}$.

The space $\left(\mathcal{C}_{a a a},\|\cdot\|_{\infty}\right)$ is complete and it is a consequence of point $(6)$.

Corollary 1. The space $\left(\mathcal{C}_{a a a},\|\cdot\|_{\infty}\right)$ is a Banach subalgebra of $\mathcal{C}_{b}$.

We have the following results on the derivative and the primitive.

Proposition 4. The following is true:

(1) Let $f=\left(f_{a a}+f_{c o r}\right) \in \mathcal{C}_{a a a}$ be such that $f^{\prime}$ exists and is uniformly continuous on $\mathbb{J}$, then there exists $\phi=(g+h) \in \mathcal{C}_{a a a}$, such that $\phi=f^{\prime}$ on $\mathbb{J},\left(f_{a a}\right)^{\prime}=g$ on $\mathbb{R}$ and $\left(f_{\text {cor }}\right)^{\prime}=h$ on $\mathbb{J}$.

(2) Let $f=\left(f_{a a}+f_{\text {cor }}\right) \in \mathcal{C}_{a a a}$ be such that $f$ is uniformly continuous on $\mathbb{J}$, then there exists $F \in$ $\mathcal{C}_{a a a}$ being a primitive of $f$ on $\mathbb{J}$ if and only if $\int_{0}^{x} f_{a a}(t) d t$ is bounded on $\mathbb{R}, \int_{0}^{x} f_{\text {cor }}(t) d t$ is bounded on $\mathbb{J}$, and $\int_{0}^{+\infty} f_{\text {cor }}(t) d t<\infty$.

P r o o f. (1) Let $\left(\sigma_{m}\right)_{m \in \mathbb{N}} \subset \mathbb{J}$ converging to zero and define the sequence $\left(\phi_{m}\right)_{m \in \mathbb{N}} \subset \mathcal{C}_{a a a}$ by

$$
\begin{aligned}
\phi_{m}(x) & =\frac{f\left(x+\sigma_{m}\right)-f(x)}{\sigma_{m}}, \quad x \in \mathbb{R} \\
& =\int_{0}^{1} f^{\prime}\left(x+\theta \sigma_{m}\right) d \theta, \quad x \in \mathbb{J} .
\end{aligned}
$$

then the sequence $\left(\phi_{m}\right)_{m}$ converges uniformly to $f^{\prime}$ on $\mathbb{J}$ and for $x \in \mathbb{J}$,

$$
\phi_{m}(x)=\phi_{m, a a}(x)+\phi_{m, c o r}(x),
$$

where

$$
\phi_{m, a a}(x):=\frac{f_{a a}\left(x+\sigma_{m}\right)-f_{a a}(x)}{\sigma_{m}}, \quad \phi_{m, c o r}(x):=\frac{f_{c o r}\left(x+\sigma_{m}\right)-f_{c o r}(x)}{\sigma_{m}} .
$$

By (1), there exists $\phi=(g+h) \in \mathcal{C}_{a a a}$, such that $\phi=f^{\prime}$ on $\mathbb{J}, g \in \mathcal{C}_{a a}$ is the uniform limit of $\left(\phi_{m, a a}\right)_{m}$ on $\mathbb{R}$ and $h \in \mathcal{C}_{+, 0}$ is the uniform limit of $\left(\phi_{m, c o r}\right)_{m}$ on $\mathbb{J}$. Hence $\left(f_{a a}\right)^{\prime}:=\lim _{m \rightarrow+\infty} \phi_{m, a a}=g$ on $\mathbb{R}$ and $\left(f_{\text {cor }}\right)^{\prime}:=\lim _{m \rightarrow+\infty} \phi_{m, \text { cor }}=h$ on $\mathbb{J}$.

(2) If $F=\left(F_{a a}+F_{c o r}\right) \in \mathcal{C}_{a a a}$ is a primitive of $f$ on $\mathbb{J}$, then $F^{\prime}=f$ is uniformly continuous on $\mathbb{J}$. By $(2),\left(F_{a a}\right)^{\prime} \in \mathcal{C}_{a a}$, there exists $h \in \mathcal{C}_{+, 0}$ such that $\left(F_{c o r}\right)^{\prime}=h$ on $\mathbb{J}$ and $F^{\prime}=\left(F_{a a}\right)^{\prime}+\left(F_{c o r}\right)^{\prime}$ on $\mathbb{J}$. Consequently, by Proposition 1-(6) and Proposition 2-(5), we obtain the result. Conversely, as $\int_{0}^{+\infty} f_{\text {cor }}(t) d t<\infty$ and $\int_{0}^{x} f_{c o r}(t) d t$ is bounded on $\mathbb{J}$, by Proposition $1-(6)$, there exits $H \in \mathcal{C}_{+, 0}$ which is a primitive on $\mathbb{J}$ of $f_{c o r}$ and as $\int_{0}^{x} f_{a a}(t) d t$ is bounded on $\mathbb{R}$, by Proposition 2-(5), there exits $G \in \mathcal{C}_{a a}$ which is a primitive on $\mathbb{R}$ of $f_{a a}$, so $F:=G+H$ is a primitive on $\mathbb{J}$ of $f$.

Corollary 2. Let $f=\left(f_{a a}+f_{c o r}\right) \in \mathcal{C}_{a a a}$ such that $f^{\prime}$ exists and is uniformly continuous on $\mathbb{R}$, then $f^{\prime}=(g+h) \in \mathcal{C}_{a a a}$, where $\left(f_{a a}\right)^{\prime}=g$ on $\mathbb{R}$ and $\left(f_{\text {cor }}\right)^{\prime}=h$ on $\mathbb{J}$. 


\section{Smooth asymptotically almost automorphic functions}

Let $\mathcal{E}(\mathbb{I})$ be the space of infinitely derivable functions on $\mathbb{I}=\mathbb{R}$ or $\mathbb{J}$, and $p \in[1,+\infty]$, the space

$$
\mathcal{D}_{L^{p}}(\mathbb{I}):=\left\{\varphi \in \mathcal{E}(\mathbb{I}): \forall j \in \mathbb{Z}_{+}, \varphi^{(j)} \in L^{p}(\mathbb{I})\right\}
$$

endowed with the topology defined by the family of seminorms

$$
|\varphi|_{k, p, \mathbb{I}}:=\sum_{j \leq k}\left\|\varphi^{(i)}\right\|_{L^{p}(\mathbb{I})}, \quad k \in \mathbb{Z}_{+},
$$

is a Fréchet subalgebra of $\mathcal{E}(\mathbb{I})$. The spaces $\mathcal{D}_{L^{p}}(\mathbb{I})$ studied in [13] are connected with the classical Sobolev spaces $W^{m, p}(\mathbb{I})$, see $[15]$. We denote $\mathcal{B}(\mathbb{I}):=\mathcal{D}_{L^{\infty}}(\mathbb{I})$. Let $\dot{\mathcal{B}}$ be the closure in $\mathcal{B}:=\mathcal{B}(\mathbb{R})$ of the space $\mathcal{D}$ of smooth functions with compact support.

Remark 4. By the definition $\varphi \in \mathcal{B}(\mathbb{J})$ requires that $\lim _{x \rightarrow 0} \varphi^{(j)}(x)$ exists $\forall j \in \mathbb{Z}_{+}$.

Let $\mathcal{B}_{+, 0}$ be the space of smooth functions vanishing at infinity, i.e.

$$
\mathcal{B}_{+, 0}:=\left\{\varphi \in \mathcal{E}(\mathbb{R}): \forall j \in \mathbb{Z}_{+}, \varphi^{(j)} \in \mathcal{C}_{+, 0}\right\} .
$$

We endow $\mathcal{B}_{+, 0}$ with the topology induced by $\mathcal{B}$.

Proposition 5. The following is true:

(1) The space $\mathcal{B}_{+, 0}$ is a Fréchet subalgebra of $\mathcal{B}$.

(2) $\tau_{\omega} \mathcal{B}_{+, 0} \subset \mathcal{B}_{+, 0}, \forall \omega \in \mathbb{R}$.

(3) $\mathcal{B}_{+, 0} \times \mathcal{B} \subset \mathcal{B}_{+, 0}$.

(4) $\mathcal{B}_{+, 0} * L^{1} \subset \mathcal{B}_{+, 0}$.

(5) $\mathcal{B}_{+, 0}=\mathcal{C}_{+, 0} \cap \mathcal{B}$.

(6) There exists $H \in \mathcal{B}_{+, 0}$ which is a primitive on $\mathbb{J}$ of $h \in \mathcal{B}_{+, 0}$ if and only if $\int_{0}^{x} h(t) d t$ is bounded on $\mathbb{J}$ and $\int_{0}^{+\infty} h(t) d t<\infty$.

P r o o f. (1) It is easy to see that $\mathcal{B}_{+, 0}$ is an algebra and since $\mathcal{B}$ is complete, it suffices to show that $\mathcal{B}_{+, 0}$ is closed. Let $\left(h_{m}\right)_{m \in \mathbb{N}}$ be a sequence of $\mathcal{B}_{+, 0}$ that converges to $h \in \mathcal{B}$, i.e. $\forall i \in \mathbb{Z}_{+},\left(h_{m}^{(i)}\right)_{m}$ converges uniformly on $\mathbb{R}$ to $h^{(i)}$. By Proposition $1-(1), h^{(i)} \in \mathcal{C}_{+, 0}, \forall i \in \mathbb{Z}_{+}$, i.e. $h \in \mathcal{B}_{+, 0}$.

(2) This inclusion is obvious.

(3) If $\varphi \in \mathcal{B}$ and $h \in \mathcal{B}_{+, 0}$, then by Leibniz's formula and Proposition $1-(3), \forall i \in \mathbb{Z}_{+}$, $(h \varphi)^{(i)} \in \mathcal{C}_{+, 0}$.

(4) Let $\psi \in L^{1}$ and $h \in \mathcal{B}_{+, 0}$, then by Proposition 1-(4), $\forall i \in \mathbb{Z}_{+},(h * \psi)^{(i)}=h^{(i)} * \psi \in \mathcal{C}_{+, 0}$.

(5) It is clear that $\mathcal{B}_{+, 0} \subset \mathcal{C}_{+, 0} \cap \mathcal{B}$. Conversely, if $h \in \mathcal{C}_{+, 0} \cap \mathcal{B}$, then $h^{\prime}$ is uniformly continuous on $\mathbb{R}$, so by Remark $2, h^{\prime} \in \mathcal{C}_{+, 0}$. By repeating this to all derivatives, we obtain that $h \in \mathcal{B}_{+, 0}$.

(6) The necessity is a consequence of Proposition 1-(6). To prove the sufficiency we need the following preliminary result on extension operators, it can be obtained from [14]: there exist 
two sequences of real numbers $\left(a_{l}\right)_{l \in \mathbb{Z}_{+}}$and $\left(b_{l}\right)_{l \in \mathbb{Z}_{+}}$such that $b_{l} \leq 0, \forall l \in \mathbb{Z}_{+}$, and the operator $E: \mathcal{B}(\mathbb{J}) \rightarrow \mathcal{B}(\mathbb{R})$ defined by

$$
E f(x):=\left\{\begin{array}{lll}
f(x) & \text { if } \quad x \geq 0 \\
\sum_{l=0}^{+\infty} a_{l} f\left(b_{l} x\right) & \text { if } \quad x<0
\end{array}\right.
$$

is linear and continuous. Suppose that $\int_{0}^{x} h(t) d t$ is bounded on $\mathbb{J}$ and $\int_{0}^{+\infty} h(t) d t<\infty$. By Proposition 1-(6), there exits $E \in \mathcal{C}_{+, 0}$ such that $E^{\prime}=h$ on $\mathbb{J}$, so $E$ is a smooth function on $\mathbb{J}$ such that $\forall i \in \mathbb{Z}_{+}, E^{(i)}$ is bounded on $\mathbb{J}$, i.e. $E \in \mathcal{B}(\mathbb{J})$. Due to the extension result there exists a function $H \in \mathcal{B}$ such that $H=E$ on $\mathbb{J}$. So $H \in \mathcal{B} \cap \mathcal{C}_{+, 0}=\mathcal{B}_{+, 0}$ and it is a primitive of $h$ on $\mathbb{J}$.

Recall the definition and some properties of the space of smooth almost automorphic functions, see [6] for details.

$$
\mathcal{B}_{a a}:=\left\{\varphi \in \mathcal{E}: \forall j \in \mathbb{Z}_{+}, \varphi^{(j)} \in \mathcal{C}_{a a}\right\}
$$

Proposition 6. The following is true:

(1) $\mathcal{B}_{a a}$ is a Fréchet subalgebra of $\mathcal{B}$.

(2) $\tau_{\omega} \mathcal{B}_{a a} \subset \mathcal{B}_{a a}, \forall \omega \in \mathbb{R}$.

(3) $\mathcal{B}_{a a} * L^{1} \subset \mathcal{B}_{a a}$.

(4) $\mathcal{B}_{a a}=\mathcal{C}_{a a} \cap \mathcal{B}$

(5) Let $f \in \mathcal{B}_{a a}$ and $F$ is its primitive on $\mathbb{R}$, then $F \in \mathcal{B}_{a a}$ if and only if $F$ is bounded.

We now introduce smooth asymptotically almost automorphic functions.

Definition 4. The space of smooth asymptotically almost automorphic functions is denoted and defined by

$$
\mathcal{B}_{a a a}:=\left\{\varphi \in \mathcal{E}: \forall j \in \mathbb{Z}_{+}, \varphi^{(j)} \in \mathcal{C}_{a a a}\right\} .
$$

Example 2. $\mathcal{B}_{a a} \subset \mathcal{B}_{a a a}$ and $\mathcal{B}_{+, 0} \subset \mathcal{B}_{a a a}$.

We endow $\mathcal{B}_{\text {aaa }}$ with the topology induced by $\mathcal{B}$. The following proposition is proved in the same way as Proposition 5 by using results of Propositions 3 and 4 .

Proposition 7. The following is true:

(1) The space $\mathcal{B}_{a a a}$ is a Fréchet subalgebra of $\mathcal{B}$.

(2) $\tau_{\omega} \mathcal{B}_{\text {aaa }} \subset \mathcal{B}_{\text {aaa }}, \forall \omega \in \mathbb{R}$.

(3) $\mathcal{B}_{a a a} \times \mathcal{B}_{a a} \subset \mathcal{B}_{a a a}$

(4) $\mathcal{B}_{\text {aaa }} * L^{1} \subset \mathcal{B}_{\text {aaa }}$.

(5) $\mathcal{B}_{\text {aaa }}=\mathcal{C}_{\text {aaa }} \cap \mathcal{B}$. 
(6) There exists $F \in \mathcal{B}_{\text {aaa }}$ being a primitive on $\mathbb{J}$ of $f \in \mathcal{B}_{\text {aaa }}$ if and only if $\int_{0}^{x} f_{a a}(t) d t$ is bounded on $\mathbb{R}, \int_{0}^{x} f_{\text {cor }}(t) d t$ is bounded on $\mathbb{J}, \quad \int_{0}^{+\infty} f_{\text {cor }}(t) d t<\infty$.

Remark 5. $\mathcal{B}_{\text {aaa }} \subsetneq \mathcal{C}_{\text {aaa }} \cap \mathcal{E}$.

We have the following result needed in the sequel.

Proposition 8. Let $f \in \mathcal{B}_{a a a}$, i.e. $f=f_{a a}+f_{\text {cor }}$ and for $i \in \mathbb{N}, f^{(i)}=f_{a a, i}+f_{c o r, i}$ on $\mathbb{J}$. Then $f_{a a, i}=\left(f_{a a}\right)^{(i)}$ on $\mathbb{R}$ and $f_{\text {cor }, i}=\left(f_{\text {cor }}\right)^{(i)}$ on $\mathbb{J}$.

P r o o f. If $f \in \mathcal{B}_{a a a}$, then $f^{\prime}$ is uniformly continuous on $\mathbb{R}$ and by Proposition 4-(2), we have $f^{\prime}=\left(f_{a a}\right)^{\prime}+h$ on $\mathbb{J}$, where $\left(f_{a a}\right)^{\prime} \in \mathcal{C}_{a a}, h \in \mathcal{C}_{+, 0}$ and $\left(f_{c o r}\right)^{\prime}=h$ on $\mathbb{J}$. By hypothesis, $f^{\prime}=f_{a a, 1}+f_{c o r, 1}$ on $\mathbb{J}$ and since the decomposition of an asymptotically almost automorphic function is unique, then $\left(f_{a a}\right)^{\prime}=f_{a a, 1}$ on $\mathbb{R}$ and $\left(f_{c o r}\right)^{\prime}=f_{c o r, 1}$ on $\mathbb{J}$. By repeating this to all derivative, we obtain the desired result.

In order to prove the main result on linear neutral difference differential equations in the framework of asymptotically almost automorphic distributions, we need the following characterization of the space $\mathcal{B}_{a a}$.

Proposition 9. Let $g \in \mathcal{E}$, the following statements are equivalent:

(1) $g \in \mathcal{B}_{a a}$.

(2) For every sequence $\left(\rho_{m}\right)_{m \in \mathbb{N}} \subset \mathbb{R}$ there exist a subsequence $\left(\rho_{m_{k}}\right)_{k}$ and $\tilde{g} \in \mathcal{B}$ such that for all $x \in \mathbb{R}$ and $i \in \mathbb{Z}_{+}$, we have

$$
\tilde{g}^{(i)}(x)=\lim _{k \rightarrow+\infty} g^{(i)}\left(x+\rho_{m_{k}}\right) \text { and } \lim _{k \rightarrow+\infty} \tilde{g}^{(i)}\left(x-\rho_{m_{k}}\right)=g^{(i)}(x) .
$$

P r o o f. (1) $\Rightarrow(2)$ Let $g \in \mathcal{B}_{a a}$, so $\forall i \in \mathbb{Z}_{+}, \forall\left(\rho_{m}\right)_{m \in \mathbb{N}} \subset \mathbb{R}, \exists\left(\rho_{m_{i, k}}\right)_{k} \subset\left(\rho_{m}\right)_{m}, \exists\left(\tilde{g}_{i}\right)_{i} \subset L^{\infty}$ such that $\forall x \in \mathbb{R}$,

$$
\lim _{k \rightarrow+\infty} g^{(i)}\left(x+\rho_{m_{i, k}}\right)=: \tilde{g}_{i}(x) \text { and } \lim _{k \rightarrow+\infty} \tilde{g}_{i}\left(x-\rho_{m_{i, k}}\right)=g^{(i)}(x) .
$$

There exist subsequences $\left(\rho_{m_{n, k}}\right)_{k}, n \in \mathbb{Z}_{+}$, of the sequence $\left(\rho_{m}\right)_{m}$ such that

$$
\forall i \leq n, \lim _{k \rightarrow+\infty} g^{(i)}\left(x+\rho_{m_{n, k}}\right)=\tilde{g}_{i}(x), \quad \forall x \in \mathbb{R} .
$$

Indeed, the proof is done by induction, if $g \in \mathcal{C}_{a a}$ it is clear that (3.2) holds for $n=0$. Now, let $n \in \mathbb{N}$ such that $(3.2)$ holds. As $g^{(n+1)} \in \mathcal{C}_{a a}$, there exists a subsequence $\left(\rho_{m_{(n+1), k}}\right)_{k}$ of $\left(\rho_{m_{n, k}}\right)_{k}$ and $\tilde{g}_{n+1} \in L^{\infty}$ such that $\forall x \in \mathbb{R}$,

$$
\tilde{g}_{n+1}(x):=\lim _{k \rightarrow+\infty} g^{(n+1)}\left(x+\rho_{m_{(n+1), k}}\right) .
$$

Furthermore, as $\forall i \leq n, \forall x \in \mathbb{R}$, the subsequence $\left(g^{(i)}\left(x+\rho_{m_{(n+1), k}}\right)\right)_{k}$ is extracted from $\left(g^{(i)}\left(x+\rho_{m_{n, k}}\right)\right)_{k}$ then

$$
\lim _{k \rightarrow+\infty} g^{(i)}\left(x+\rho_{m_{(n+1), k}}\right)=\tilde{g}_{i}(x), \quad \forall x \in \mathbb{R} .
$$


By construction, $\forall k, i, \in \mathbb{Z}_{+}, m_{i, k} \leq m_{(i+1), k}$ and since $k \longmapsto m_{(i+1), k}$ is strictly increasing from $\mathbb{N}$ to $\mathbb{N}$, then in particular we have $m_{i, i} \leq m_{(i+1), i}<m_{(i+1),(i+1)}, \forall i \in \mathbb{Z}_{+}$. This gives that the map $k \longmapsto m_{k, k}$ is strictly increasing from $\mathbb{N}$ to $\mathbb{N}$. The sequence $\left(\rho_{m_{k, k}}\right)_{k}$, which we denote by $\left(\rho_{m_{k}}\right)_{k}$, is extracted from the subsequences $\left(\rho_{m_{i, k}}\right)_{k}, i \in \mathbb{Z}_{+}$, which is in fact extracted from the sequence $\left(\rho_{m}\right)_{m}$. Consequently,

$$
\lim _{k \rightarrow+\infty} g^{(i)}\left(x+\rho_{m_{k}}\right)=\tilde{g}_{i}(x) \quad \text { exists } \quad \forall x \in \mathbb{R}, \quad \forall i \in \mathbb{Z}_{+} .
$$

With the same steps we have that

$$
\lim _{k \rightarrow+\infty} \tilde{g}_{i}\left(x-\rho_{m_{k}}\right)=g^{(i)}(x), \quad \forall x \in \mathbb{R}, \quad \forall i \in \mathbb{Z}_{+} .
$$

Let $\left(\sigma_{n}\right)_{n \in \mathbb{N}} \subset \mathbb{J}$ converging to zero and consider the sequence of functions $\left(\phi_{n, k}\right)_{n, k \in \mathbb{N}}$ defined on $\mathbb{R}$ by the equality

$$
\phi_{n, k}(\cdot)=\frac{g\left(\cdot+\rho_{m_{k}}+\sigma_{n}\right)-g\left(\cdot+\rho_{m_{k}}\right)}{\sigma_{n}}=\int_{0}^{1} g^{\prime}\left(\cdot+\rho_{m_{k}}+\theta \sigma_{n}\right) d \theta .
$$

Since $g \in \mathcal{B}_{a a} \subset \mathcal{B}$, then $g^{\prime}$ is bounded and uniformly continuous on $\mathbb{R}$, so

$$
\lim _{k \rightarrow+\infty} \lim _{n \rightarrow+\infty} \int_{0}^{1} g^{\prime}\left(\cdot+\rho_{m_{k}}+\theta \sigma_{n}\right) d \theta=\lim _{n \rightarrow+\infty} \lim _{k \rightarrow+\infty} \int_{0}^{1} g^{\prime}\left(\cdot+\rho_{m_{k}}+\theta \sigma_{n}\right) d \theta .
$$

Consequently, $\forall x \in \mathbb{R}, \lim _{k \rightarrow+\infty} \lim _{n \rightarrow+\infty} \phi_{n, k}(x)=\lim _{n \rightarrow+\infty} \lim _{k \rightarrow+\infty} \phi_{n, k}(x)$ which gives that $\forall x \in \mathbb{R}$,

$$
\tilde{g}_{1}(x)=\lim _{k \rightarrow+\infty} \lim _{n \rightarrow+\infty} \phi_{n, k}(x)=\lim _{n \rightarrow+\infty} \lim _{k \rightarrow+\infty} \phi_{n, k}(x):=\tilde{g}_{0}^{\prime}(x) .
$$

By iterating to all derivatives, we obtain that $\tilde{g}_{0} \in \mathcal{E}$ and $\tilde{g}_{0}^{(i)}=\tilde{g}_{i} \in L^{\infty}, \forall i \in \mathbb{Z}_{+}$, i.e. $\tilde{g}_{0} \in \mathcal{B}$ such that relations (3.1) hold.

$(2) \Rightarrow(1)$ is obvious.

\section{Asymptotically almost automorphic distributions}

The space of $L^{p}$-distributions, denoted by $\mathcal{D}_{L^{p}}^{\prime}$, is the topological dual of $\mathcal{D}_{L^{q}}$, where $1 / p+1 / q=1$. The topological dual of $\dot{\mathcal{B}}$ is denoted by $\mathcal{D}_{L^{1}}^{\prime}$. The space of bounded distributions $\mathcal{D}_{L^{\infty}}^{\prime}$ is denoted by $\mathcal{B}^{\prime}$. The translate $\tau_{\omega} T, \omega \in \mathbb{R}$, of a distribution $T \in \mathcal{D}^{\prime}$ is defined by $\left\langle\tau_{\omega} T, \varphi\right\rangle=\left\langle T, \tau_{-\omega} \varphi\right\rangle, \forall \varphi \in \mathcal{D}$.

Definition 5. By $\mathcal{B}_{+, 0}^{\prime}$ we denote the space of distributions $Q \in \mathcal{B}^{\prime}$ vanishing at infinity, i.e. satisfying

$$
\lim _{\omega \rightarrow+\infty}\left\langle\tau_{\omega} Q, \varphi\right\rangle=0, \quad \forall \varphi \in \mathcal{D}
$$

We have the following characterizations of $\mathcal{B}_{+, 0}^{\prime}$, see [7].

Theorem 1. Let $Q \in \mathcal{B}^{\prime}$, the following assertions are equivalent:

(1) $Q \in \mathcal{B}_{+, 0}^{\prime}$

(2) $Q * \varphi \in \mathcal{C}_{+, 0}, \forall \varphi \in \mathcal{D}$. 
(3) $\exists k \in \mathbb{Z}_{+}$and $h_{j} \in \mathcal{C}_{+, 0}, 0 \leq j \leq k$, such that $Q=\sum_{j=0}^{k} h_{j}^{(j)}$.

We study some properties of the space $\mathcal{B}_{+, 0}^{\prime}$.

Proposition 10. The following is true:

(1) If $Q \in \mathcal{B}_{+, 0}^{\prime}$, then $Q^{(i)} \in \mathcal{B}_{+, 0}^{\prime}, \forall i \in \mathbb{Z}_{+}$.

(2) $\tau_{\omega} \mathcal{B}_{+, 0}^{\prime} \subset \mathcal{B}_{+, 0}^{\prime}, \forall \omega \in \mathbb{R}$.

(3) $\mathcal{B}_{+, 0}^{\prime} \times \mathcal{B} \subset \mathcal{B}_{+, 0}^{\prime}$.

(4) $\mathcal{B}_{+, 0}^{\prime} * \mathcal{D}_{L^{1}}^{\prime} \subset \mathcal{B}_{+, 0}^{\prime}$.

(5) Let $Q \in \mathcal{B}^{\prime}$, then $Q \in \mathcal{B}_{+, 0}^{\prime}$ if and only if there exists a sequence $\left(\varphi_{m}\right)_{m \in \mathbb{N}} \subset \mathcal{B}_{+, 0}$ converging to $Q$ in $\mathcal{B}^{\prime}$.

P r o o f. (1) and (2) are obvious.

(3) Let $\varphi \in \mathcal{B}$ and $Q \in \mathcal{B}_{+, 0}^{\prime}$, then by Theorem 1-(3), there exist $\left(h_{i}\right)_{i \leq k} \subset \mathcal{C}_{+, 0}$, such that $Q=\sum_{i=0}^{k} h_{i}^{(i)}$. So

$$
\varphi Q=\sum_{i=0}^{k} \varphi h_{i}^{(i)}=\sum_{i=0}^{k} \sum_{j=0}^{i}(-1)^{j}\left(\begin{array}{l}
i \\
j
\end{array}\right)\left(\varphi^{(j)} h_{i}\right)^{(i-j)} .
$$

By Proposition 1-(3), $\varphi^{(j)} h_{i} \in \mathcal{C}_{+, 0}$, hence $\varphi Q \in \mathcal{B}_{+, 0}^{\prime}$.

(4) Let $Q \in \mathcal{B}_{+, 0}^{\prime}$, then there exists $\left(h_{i}\right)_{i \leq k} \subset \mathcal{C}_{+, 0}$ such that $Q=\sum_{i=0}^{k} h_{i}^{(i)}$, and let $S \in \mathcal{D}_{L^{1}}^{\prime}$, by $\left[13\right.$, Theorem $X X V$, Section 8 , Chapter VI], there exist $\left(\psi_{j}\right)_{j \leq m} \subset L^{1}$ such that $S=\sum_{j=0}^{m} \psi_{j}^{(j)}$. Thus

$$
(Q * S)=\sum_{i=0}^{k} \sum_{j=0}^{m}\left(h_{i} * \psi_{j}\right)^{(i+j)} .
$$

By Proposition 1-(4), $h_{i} * \psi_{j} \in \mathcal{C}_{+, 0}$, hence $Q * S \in \mathcal{B}_{+, 0}^{\prime}$.

(5) Let $\left.\phi_{m}\right)_{m \in \mathbb{N}} \subset \mathcal{B}_{+, 0}$ such that $\lim _{m \rightarrow+\infty} \phi_{m}=Q$ in $\mathcal{B}^{\prime}$. For a fixed $\varphi \in \mathcal{D}$, the set

$$
U:=\left\{\tau_{-x} \check{\varphi}: x \in \mathbb{R}\right\}
$$

is bounded in $\mathcal{D}_{L^{1}}$, so

$$
\begin{aligned}
\sup _{x \in \mathbb{R}}\left|\left(\phi_{m} * \varphi\right)(x)-(Q * \varphi)(x)\right| & =\sup _{x \in \mathbb{R}}\left|\left\langle\phi_{m}-Q, \tau_{-x} \check{\varphi}\right\rangle\right|, \\
& =\sup _{\psi \in U}\left|\left\langle\phi_{m}-Q, \psi\right\rangle\right| \underset{m \rightarrow+\infty}{\longrightarrow} 0,
\end{aligned}
$$

i.e. $\left(\phi_{m} * \varphi\right)_{m \in \mathbb{N}} \subset \mathcal{C}_{+, 0}$ is uniformly convergent to $(Q * \varphi)$. By Proposition 1-(1), $Q * \varphi \in \mathcal{C}_{+, 0}, \forall \varphi \in \mathcal{D}$, and by Theorem 1, we obtain $Q \in \mathcal{B}_{+, 0}^{\prime}$.

Conversely, let $Q \in \mathcal{B}_{+, 0}^{\prime}$ and take a sequence of positive test functions $\left(\theta_{m}\right)_{m \in \mathbb{N}}$ such that

$$
\operatorname{supp} \theta_{m} \subset\left[0, \frac{1}{m}\right] \text { and } \int_{\mathbb{R}} \theta_{m}(x) d x=1 .
$$


Define $\phi_{m}:=\theta_{m} * Q \in \mathcal{B}_{+, 0}$, we have

$$
\left\langle\phi_{m}-Q, \varphi\right\rangle=\left\langle Q, \check{\theta}_{m} * \varphi-\varphi\right\rangle,
$$

and there exist $l \in \mathbb{Z}_{+}, C>0$ such that

$$
\left|\left\langle Q, \check{\theta}_{m} * \varphi-\varphi\right\rangle\right| \leq C\left|\check{\theta}_{m} * \varphi-\varphi\right|_{l, 1}, \quad \forall \varphi \in \mathcal{D}_{L^{1}} .
$$

By Minkowski's inequality and the mean value theorem we obtain for $t \in] 0,1[$,

$$
\begin{aligned}
& \left\|\left(\check{\theta}_{m} * \varphi\right)^{(i)}-\varphi^{(i)}\right\|_{L^{1}} \leq \int_{0}^{1 / m} \check{\theta}_{m}(y)\left(\int_{\mathbb{R}}\left|y \| \varphi^{(i+1)}(x+t y)\right| d x\right) d y \\
& \leq \int_{0}^{1 / m}|y| \check{\theta}_{m}(y)\left(\int_{\mathbb{R}}\left|\varphi^{(i+1)}(z)\right| d z\right) d y \leq \frac{1}{m}\left\|\varphi^{(i+1)}\right\|_{L^{1}}\left\|\check{\theta}_{m}\right\|_{L^{1}},
\end{aligned}
$$

so

$$
\left|\check{\theta}_{m} * \varphi-\varphi\right|_{l, 1} \leq \frac{1}{m}|\varphi|_{l+1,1}, \quad \forall \varphi \in \mathcal{D}_{L^{1}}
$$

Let $U$ be a bounded set of $\mathcal{D}_{L^{1}}$ and $\varphi \in U$, then $\exists M>0$ such that

$$
\sup _{\varphi \in U}\left|\check{\theta}_{m} * \varphi-\varphi\right|_{l, 1} \leq \frac{M}{m} \underset{m \rightarrow+\infty}{\longrightarrow} 0
$$

which gives $\theta_{m} \rightarrow Q$ in $\mathcal{B}^{\prime}$.

We recall the definition, characterizations and some properties of almost automorphic distributions, see [6].

Definition 6. A distribution $T \in \mathcal{B}^{\prime}$ is said almost automorphic if it satisfies one of the following equivalent conditions:

(1) $T * \varphi \in \mathcal{C}_{a a}, \forall \varphi \in \mathcal{D}$.

(2) $\exists k \in \mathbb{Z}_{+}$and $g_{j} \in \mathcal{C}_{a a}, 0 \leq j \leq k$, such that $T=\sum_{i=0}^{k} g_{i}^{(i)}$.

(3) For every sequence $\left(s_{m}\right)_{m \in \mathbb{N}} \subset \mathbb{R}$, there is a subsequence $\left(s_{m_{k}}\right)_{k}$ such that

$$
S:=\lim _{k \rightarrow+\infty} \tau_{s_{m_{k}}} T \text { exists in } \mathcal{D}^{\prime},
$$

and

$$
\lim _{k \rightarrow+\infty} \tau_{-s_{m_{k}}} S=T \quad \text { in } \quad \mathcal{D}^{\prime}
$$

(4) There exists a sequence $\left(\varphi_{m}\right)_{m \in \mathbb{N}} \subset \mathcal{B}_{a a}$ converging to $T$ in $\mathcal{B}^{\prime}$.

We denote by $\mathcal{B}_{a a}^{\prime}$ the space of almost automorphic distributions defined on $\mathbb{R}$.

Proposition 11. The following is true:

(1) If $T \in \mathcal{B}_{a a}^{\prime}$, then $T^{(i)} \in \mathcal{B}_{a a}^{\prime}, \forall i \in \mathbb{Z}_{+}$. 
(2) $\tau_{\omega} \mathcal{B}_{a a}^{\prime} \subset \mathcal{B}_{a a}^{\prime}, \forall \omega \in \mathbb{R}$

(3) $\mathcal{B}_{a a}^{\prime} \times \mathcal{B}_{a a} \subset \mathcal{B}_{a a}^{\prime}$.

(4) $\mathcal{B}_{a a}^{\prime} * \mathcal{D}_{L^{1}}^{\prime} \subset \mathcal{B}_{a a}^{\prime}$.

(5) $\mathcal{B}_{a a}^{\prime} \cap \mathcal{B}_{+, 0}^{\prime}=\{0\}$

We now give the definition of asymptotically almost automorphic distributions.

Definition 7. A distribution $T \in \mathcal{B}^{\prime}$ is said asymptotically almost automorphic if there exist $P \in \mathcal{B}_{a a}^{\prime}$ and $Q \in \mathcal{B}_{+, 0}^{\prime}$ such that $T=P+Q$ on $\mathbb{J}$. We denote by $\mathcal{B}_{a a a}^{\prime}$ the space of asymptotically almost automorphic distributions.

Remark 6 . The equality $T=P+Q$ on $\mathbb{J}$ means that $\forall \varphi \in \mathcal{D}_{+},\langle T, \varphi\rangle=\langle P, \varphi\rangle+\langle Q, \varphi\rangle$, where $\mathcal{D}_{+}:=\{\varphi \in \mathcal{D}: \operatorname{supp} \varphi \subset \mathbb{J}\}$.

Proposition 12. The decomposition of an asymptotically almost automorphic distribution is unique on $\mathbb{J}$.

P r o o f. Let $P_{1}, P_{2} \in \mathcal{B}_{a a}^{\prime}$ and $Q_{1}, Q_{2} \in \mathcal{B}_{+, 0}^{\prime}$ such that $T=P_{1}+Q_{1}=P_{2}+Q_{2}$ on $\mathbb{J}$, then we obtain that $P_{1}-P_{2} \in \mathcal{B}_{+, 0}^{\prime}$, by Proposition 11-(5), $P_{1}-P_{2}=0$. Hence $Q_{1}=Q_{2}$ on $\mathbb{J}$.

Notation 1. If $T \in \mathcal{B}_{\text {aaa }}^{\prime}$ and $T=P+Q$ on $\mathbb{J}$, we call $P$ the principal term and $R$ the corrective term of $T$ and we denote them respectively by $T_{a a}$ and $T_{c o r}$. This is summarized by the notation $T=\left(T_{a a}+T_{c o r}\right) \in \mathcal{B}_{a a a}^{\prime}$.

\section{Example 3.}

1. $\mathcal{C}_{a a a} \subset \mathcal{B}_{\text {aaa }}^{\prime}$

2. $\mathcal{B}_{a a}^{\prime} \subset \mathcal{B}_{a a a}^{\prime}$.

3. $\mathcal{B}_{+, 0}^{\prime} \subset \mathcal{B}_{\text {aaa }}^{\prime}$

4. $\mathcal{B}_{\text {aap }}^{\prime} \mp \mathcal{B}_{\text {aaa }}^{\prime}$, where $\mathcal{B}_{\text {aap }}^{\prime}$ is the space of asymptotically almost periodic distributions of [7].

The following results characterize asymptotically almost automorphic distributions.

Theorem 2. Let $T \in \mathcal{B}^{\prime}$, the following assertions are equivalent:

(1) $T \in \mathcal{B}_{\text {aaa }}^{\prime}$

(2) $\exists\left(\theta_{m}\right)_{m \in \mathbb{N}} \subset \mathcal{B}_{\text {aaa }}$ such that $\lim _{n \rightarrow+\infty} \theta_{m}=T$ in $\mathcal{B}^{\prime}$.

(3) $T * \varphi \in \mathcal{C}_{a a a}, \forall \varphi \in \mathcal{D}$.

(4) $\exists k \in \mathbb{Z}_{+}$and $f_{j} \in \mathcal{C}_{a a a}, 0 \leq j \leq k$, such that $T=\sum_{j=0}^{k} f_{j}^{(j)}$. 
P r o o f. (1) $\Rightarrow(2)$ Let $T \in \mathcal{B}_{a a a}^{\prime}$, by definition $T=T_{a a}+T_{c o r}$ on $\mathbb{J}$. By the characterization of $\mathcal{B}_{a a}^{\prime}$ there exists $\left(\varphi_{m}\right)_{m \in \mathbb{N}} \subset \mathcal{B}_{a a}$ such that $\lim _{m \rightarrow+\infty} \varphi_{m}=T_{a a}$ in $\mathcal{B}^{\prime}$. It is easy to prove that $T-T_{a a} \in \mathcal{B}_{+, 0}^{\prime}$, so by Proposition 10-(5) there exists $\left(\psi_{m}\right)_{m \in \mathbb{N}} \subset \mathcal{B}_{+, 0}$ such that $\lim _{m \rightarrow+\infty} \psi_{m}=$ $T-T_{a a}$ in $\mathcal{B}^{\prime}$. Set $\theta_{m}:=\varphi_{m}+\psi_{m}, m \in \mathbb{N}$, then $\left(\theta_{m}\right)_{m \in \mathbb{N}} \subset \mathcal{B}_{a a a}$ and we have $T-\theta_{m}=\left(T-T_{a a}\right)-$ $\psi_{m}+\left(T_{a a}-\varphi_{m}\right)$. Hence we obtain $\lim _{n \rightarrow+\infty} \theta_{m}=T$ in $\mathcal{B}^{\prime}$.

$(2) \Rightarrow(3)$ As in the proof of Proposition 10-(5), if $\left(\phi_{m}\right)_{m \in \mathbb{N}} \subset \mathcal{B}_{a a a}$ is such that $\lim _{m \rightarrow+\infty} \phi_{m}=T$ in $\mathcal{B}^{\prime}$, then for $\forall \varphi \in \mathcal{D}$ we have

$$
\sup _{x \in \mathbb{R}}\left|\left(\phi_{m} * \varphi\right)(x)-(T * \varphi)(x)\right|=\sup _{x \in \mathbb{R}}\left|\left\langle\phi_{m}-T, \tau_{-x} \check{\varphi}\right\rangle\right| \underset{m \rightarrow+\infty}{\longrightarrow} 0 .
$$

That is $\left(\phi_{m} * \varphi\right)_{m \in \mathbb{N}} \subset \mathcal{C}_{a a a}$ converges uniformly on $\mathbb{R}$ to $(T * \varphi)$, it follows that $T * \varphi \in \mathcal{C}_{a a a}$, $\forall \varphi \in \mathcal{D}$.

$(3) \Rightarrow(4)$ For $n \in \mathbb{Z}_{+}$, consider the function

$$
E_{n}(x)=\left\{\begin{array}{cc}
\frac{x^{n-1}}{(n-1) !}, & x \geq 0 . \\
0, & x<0 .
\end{array}\right.
$$

Then $E_{n} \in \mathcal{C}^{n-2}, \operatorname{supp} E_{n} \subset \mathbb{J}$ and $E_{n}^{(n)}=\delta$. Take a function $\gamma \in \mathcal{D}$ such that $\gamma=1$ in the neighborhood of 0 , a direct calculus gives $\left(\gamma E_{n}\right)^{(n)}=\delta+\zeta_{n}$, where

$$
\zeta_{n}=\sum_{k=0}^{n-1}\left(\begin{array}{l}
n \\
k
\end{array}\right) \gamma^{(n-k)} E_{n}^{(k)} \in \mathcal{D} .
$$

As $T \in \mathcal{B}^{\prime}$, we have

$$
T=\left(\gamma E_{n} * T\right)^{(n)}-T * \zeta_{n},
$$

where $T * \zeta_{n} \in \mathcal{C}_{a a a}$. It remains to show that $\gamma E_{n} * T \in \mathcal{C}_{a a a}$ for a suitable $n$. There exist $m \in \mathbb{Z}_{+}$ and $C>0$ such that

$$
|\langle T, \psi\rangle| \leq C|\psi|_{m, 1}, \quad \forall \psi \in \mathcal{D}_{L^{1}} .
$$

Take $n=m+2$, then $\gamma E_{m+2} \in \mathcal{D}_{L^{1}}^{m}$, where

$$
\mathcal{D}_{L^{1}}^{m}:=\left\{\varphi \in \mathcal{C}^{m}: \forall j \leq m, \varphi^{(j)} \in L^{1}\right\} \text { endowed with the norm }|\cdot|_{m, 1} .
$$

We have $\mathcal{D} \hookrightarrow \mathcal{D}_{L^{1}} \hookrightarrow \mathcal{D}_{L^{1}}^{m}$ and there exists a sequence $\left(\theta_{k}\right)_{k \in \mathbb{N}} \subset \mathcal{D}$ such that $\left(\theta_{k}\right)_{k}$ converges to $\gamma E_{m+2}$ with respect to the norm $|\cdot|_{m, 1}$, so

$$
\begin{aligned}
\left|\left(T * \theta_{k}\right)(x)-\left(T * \gamma E_{m+2}\right)(x)\right| & =\left|\left\langle T, \tau_{-x} \check{\theta}_{k}-\tau_{-x}\left(\check{\gamma}_{m+2}\right)\right\rangle\right|, \\
& \leq C\left|\tau_{-x} \check{\theta}_{k}-\tau_{-x}\left(\check{\gamma}_{m+2}\right)\right|_{m, 1}, \\
& \leq C\left|\theta_{k}-\gamma E_{m+2}\right|_{m, 1},
\end{aligned}
$$

consequently,

$$
\sup _{x \in \mathbb{R}}\left|\left(T * \theta_{k}\right)(x)-\left(T * \gamma E_{m+2}\right)(x)\right| \leq C\left|\theta_{k}-\gamma E_{m+2}\right|_{m, 1} \underset{k \rightarrow+\infty}{\longrightarrow} 0 .
$$

i.e. the sequence of functions $\left(T * \theta_{k}\right)_{k \in \mathbb{N}} \subset \mathcal{C}_{a a a}$ converges uniformly on $\mathbb{R}$ to $T * \gamma E_{m+2}$, hence $T * \gamma E_{m+2} \in \mathcal{C}_{a a a}$. 
$(4) \Rightarrow(1)$ Let $T=\sum_{j=0}^{k} f_{j}^{(j)}$, where $f_{j} \in \mathcal{C}_{a a a}, j=0, \ldots, k$, so

$$
T=\sum_{j=0}^{k} f_{j, a a}^{(j)}+\sum_{j=0}^{k} f_{j, c o r}^{(j)} \quad \text { on } \quad \mathbb{J} .
$$

Then by Theorem 1 and Definition 6,P $=\sum_{j=0}^{k} f_{j, a a}^{(j)} \in \mathcal{B}_{a a}^{\prime}$ and $Q=\sum_{j=0}^{k} f_{j, c o r}^{(j)} \in \mathcal{B}_{+, 0}^{\prime}$. Therefore $T=P+Q$ on $\mathbb{J}$, i.e. $T \in \mathcal{B}_{a a a}^{\prime}$.

Remark \%. Connected with this theorem, let us quote the preprint [10]. The authors thank the referee for pointing out the recent work [11].

We have the following properties of $\mathcal{B}_{a a a}^{\prime}$.

Proposition 13. The following is true:

(1) If $T \in \mathcal{B}_{\text {aaa }}^{\prime}$, then $\forall i \in \mathbb{Z}_{+}, T^{(i)}=\left(T_{a a}^{(i)}+T_{\text {cor }}^{(i)}\right) \in \mathcal{B}_{\text {aaa }}^{\prime}$.

(2) $\tau_{\omega} \mathcal{B}_{\text {aaa }}^{\prime} \subset \mathcal{B}_{\text {aaa }}^{\prime}, \forall \omega \in \mathbb{R}_{+}$.

(3) $\mathcal{B}_{a a a}^{\prime} \times \mathcal{B}_{a a} \subset \mathcal{B}_{\text {aaa }}^{\prime}$.

(4) $\mathcal{B}_{\text {aaa }}^{\prime} * \mathcal{D}_{L^{1}}^{\prime} \subset \mathcal{B}_{a a a}^{\prime}$.

$\mathrm{P} \mathrm{r} \mathrm{o} \mathrm{o} \mathrm{f.} \mathrm{The} \mathrm{proof} \mathrm{of} \mathrm{the} \mathrm{assertions} \mathrm{(1)-(3)} \mathrm{follows} \mathrm{from} \mathrm{the} \mathrm{definition,} \mathrm{the} \mathrm{uniqueness} \mathrm{of} \mathrm{the}$ decomposition and the same properties satisfied by the space $\mathcal{B}_{a a}^{\prime}$.

(4) Let $T \in \mathcal{B}_{\text {aaa }}^{\prime}$, by the previous Theorem, there exist $\left(f_{i}\right)_{i \leq k} \subset \mathcal{C}_{a a a}$ such that $T=\sum_{i=0}^{k} f_{i}^{(i)}$. Let $S \in \mathcal{D}_{L^{1}}^{\prime}$, by [13, Theorem XXV, Section 8, Chapter VI], there exists $\left(\psi_{j}\right)_{j \leq m} \subset L^{1}$ such that $S=\sum_{j=0}^{m} \psi_{j}^{(j)}$. Thus

$$
(T * S)=\sum_{i=0}^{k} \sum_{j=0}^{m}\left(f_{i} * \psi_{j}\right)^{(i+j)} .
$$

By Proposition 7-(4), $f_{i} * \psi_{j} \in \mathcal{C}_{a a a}$. By [13, Theorem XXVI, Section 8, Chapter VI] we have $\mathcal{B}^{\prime} * \mathcal{D}_{L^{1}}^{\prime} \subset \mathcal{B}^{\prime}$, hence $S * T \in \mathcal{B}_{a a a}^{\prime}$.

\section{Linear neutral difference differential equations}

A linear neutral difference differential equation is an equation

$$
L_{\omega} u:=\sum_{i=0}^{p} \sum_{j=0}^{q} a_{i j} \frac{d^{i}}{d x^{i}} \tau_{\omega_{j}} u+K * u=f,
$$

where $\left(a_{i j}\right)_{i \leq p, j \leq q} \subset \mathcal{B}_{a a}, K \in L^{1}$ and $\omega=\left(\omega_{j}\right)_{j \leq q} \subset \mathbb{R}_{+}^{q}$.

By the properties of the space $\mathcal{B}_{\text {aaa }}^{\prime}$ it is clear that $L_{\omega} \mathcal{B}_{\text {aaa }}^{\prime} \subset \mathcal{B}_{\text {aaa }}^{\prime}$. To prove the main result of this section we need the following result. 
Lemma 1. Let $T \in \mathcal{B}^{\prime}, g, \tilde{g} \in \mathcal{B}$ and $\left(s_{m}\right)_{m \in \mathbb{N}}$ a sequence of real numbers such that

$$
\tilde{T}:=\lim _{m \rightarrow+\infty} \tau_{s_{m}} T \text { in } \mathcal{D}^{\prime},
$$

and

$$
\tilde{g}^{(j)}(x)=\lim _{n \rightarrow+\infty} \tau_{s_{m}} g^{(j)}(x), \quad \forall x \in \mathbb{R}, \quad \forall j \in \mathbb{Z}_{+},
$$

then

$$
\lim _{n \rightarrow+\infty} \tau_{s_{m}}(g T)=\tilde{g} \tilde{T} \quad \text { in } \quad \mathcal{D}^{\prime} .
$$

P r o o f. Let $\left(s_{m}\right)_{m \in \mathbb{N}}, T \in \mathcal{B}^{\prime}$ and $g, \tilde{g} \in \mathcal{B}$ such that (5.1) and (5.2) hold. As $T \in \mathcal{B}^{\prime}, \exists C>0$, $\exists l \in \mathbb{Z}_{+}$, such that

$$
|\langle T, \psi\rangle| \leq C|\psi|_{l, 1}, \quad \forall \psi \in \mathcal{D}_{L^{1}} .
$$

So $\forall \varphi \in \mathcal{D}$,

$$
\begin{aligned}
\left|\left\langle\tau_{s_{m}}(g T)-\tilde{g} \tilde{T}, \varphi\right\rangle\right| & =\left|\left\langle T, g \tau_{-s_{m}} \varphi\right\rangle-\langle\tilde{T}, \tilde{g} \varphi\rangle\right| \\
& =\left|\left\langle\tau_{s_{m}} T, \varphi \tau_{s_{m}} g\right\rangle-\langle\tilde{T}, \tilde{g} \varphi\rangle\right|, \\
& \leq\left|\left\langle\tau_{s_{m}} T-\tilde{T}, \tilde{g} \varphi\right\rangle\right|+\left|\left\langle\tau_{s_{m}} T,\left(\tau_{s_{m}} g-\tilde{g}\right) \varphi\right\rangle\right|, \\
& \leq\left|\left\langle\tau_{s_{m}} T-\tilde{T}, \tilde{g} \varphi\right\rangle\right|+C\left|\left(\tau_{s_{m}} g-\tilde{g}\right) \varphi\right|_{l, 1}, \\
& \leq\left|\left\langle\tau_{s_{m}} T-\tilde{T}, \tilde{g} \varphi\right\rangle\right|+C \sum_{i=0}^{l}\left\|\left(\left(\tau_{s_{m}} g-\tilde{g}\right) \varphi\right)^{(i)}\right\|_{L^{1}} .
\end{aligned}
$$

The lemma is proved due to (5.1) and the following estimate

$$
\left\|\left(\left(\tau_{s_{m}} g-\tilde{g}\right) \varphi\right)^{(i)}\right\|_{L^{1}} \leq \sum_{j=0}^{i}\left(\begin{array}{l}
i \\
j
\end{array}\right) \int_{\mathbb{R}}\left|g^{(j)}\left(x+s_{m}\right)-\tilde{g}^{(j)}(x) \| \varphi^{(i-j)}(x)\right| d x \underset{m \rightarrow \infty}{\longrightarrow} 0,
$$

which is due to the dominated convergence theorem.

The main result of this section is the following.

Theorem 3. Let $S \in \mathcal{B}_{\text {aaa }}^{\prime}$, the equation $L_{\omega} T=S$ has a solution $T \in \mathcal{B}_{\text {aaa }}^{\prime}$ on $\mathbb{J}$ if and only if there exist $V \in \mathcal{B}_{a a}^{\prime}$ and $W \in \mathcal{B}_{+, 0}^{\prime}$, such that

$$
L_{\omega} V=S_{a a} \quad \text { on } \quad \mathbb{R}
$$

and

$$
L_{\omega} W=S_{\text {cor }} \quad \text { on } \quad \mathbb{J} \text {. }
$$

P r o o f. Suppose that equations (5.3) and (5.4) are satisfied, then

$$
L_{\omega}(V+W)=L_{\omega} V+L_{\omega} W=S_{a a}+S_{c o r}=S \quad \text { on } \quad \mathbb{J} .
$$

So $T=V+W \in \mathcal{B}_{\text {aaa }}^{\prime}$ is a solution on $\mathbb{J}$ of $L_{\omega} T=S$.

Conversely, let $T \in \mathcal{B}_{a a a}^{\prime}$ be a solution on $\mathbb{J}$ of the equation $L_{\omega} T=S$ and let $\left(s_{m}\right)_{m \in \mathbb{N}}$ be a sequence of real numbers which converges to $+\infty$. As $S_{a a}, T_{a a} \in \mathcal{B}_{a a}^{\prime}$ and $S_{c o r}, T_{c o r} \in \mathcal{B}_{+, 0}^{\prime}$, and by Proposition 9 , there is a subsequence $\left(s_{m_{k}}\right)_{k}$ of $\left(s_{m}\right)$ converging to $+\infty$ and functions $\tilde{a}_{i j} \in \mathcal{B}$ such that $\forall x \in \mathbb{R}, \forall i \leq p, \forall j \leq q$, we have

$$
\lim _{k \rightarrow+\infty} \tau_{s_{m_{k}}} a_{i j}(x)=\tilde{a}_{i j}(x) \quad \text { exists and } \lim _{k \rightarrow+\infty} \tau_{-s_{m_{k}}} \tilde{a}_{i j}(x)=a_{i j}(x),
$$


and the following limits exist in $\mathcal{D}^{\prime}$,

$$
\begin{array}{ccc}
\lim _{k \rightarrow+\infty} \tau_{s_{m_{k}}} T_{a a}=\mathcal{V} \quad \text { and } & \lim _{k \rightarrow+\infty} \tau_{-s_{m_{k}}} \mathcal{V}=T_{a a} \\
\lim _{k \rightarrow+\infty} \tau_{s_{m_{k}}} S_{a a}=\mathcal{P} \quad \text { and } & \lim _{k \rightarrow+\infty} \tau_{-s_{m_{k}}} \mathcal{P}=S_{a a} \\
\lim _{k \rightarrow+\infty} \tau_{s_{m_{k}}} T_{\text {cor }}=0 \quad \text { and } & \lim _{k \rightarrow+\infty} \tau_{s_{m_{k}}} S_{\text {cor }}=0
\end{array}
$$

Let $\varphi \in \mathcal{D}$, we have

$$
\begin{aligned}
\left\langle\tau_{s_{m_{k}}}\left(L_{\omega} T\right), \varphi\right\rangle & =\sum_{i=0}^{p} \sum_{j=0}^{q}(-1)^{i}\left\langle T, \tau_{-\omega_{j}}\left(a_{i j} \tau_{-s_{m_{k}}} \varphi\right)^{(i)}\right\rangle+\left\langle K * \tau_{s_{m_{k}}} T, \varphi\right\rangle, \\
& =\sum_{i=0}^{p} \sum_{j=0}^{q}(-1)^{i}\left\langle\tau_{s_{m_{k}}} T, \tau_{-\omega_{j}}\left(\varphi \tau_{s_{m_{k}}} a_{i j}\right)^{(i)}\right\rangle+\left\langle K * \tau_{s_{m_{k}}} T, \varphi\right\rangle, \\
& =\sum_{i=0}^{p} \sum_{j=0}^{q}\left\langle\tau_{s_{m_{k}}} a_{i j} \tau_{\omega_{j}}\left(\tau_{s_{m_{k}}} T\right)^{(i)}, \varphi\right\rangle+\left\langle K * \tau_{s_{m_{k}}} T, \varphi\right\rangle \\
& =\left\langle L_{\omega, k}\left(\tau_{s_{m_{k}}} T\right), \varphi\right\rangle
\end{aligned}
$$

where

$$
L_{\omega, k}=\sum_{i=0}^{p} \sum_{j=0}^{q} \tau_{s_{m_{k}}} a_{i j} \frac{d^{i}}{d x^{i}} \tau_{\omega_{j}}+K * .
$$

On $\left[-s_{m_{k}},+\infty\left[\right.\right.$ we have $\tau_{s_{m_{k}}} S=\tau_{s_{m_{k}}} L_{\omega} T$, i.e.

$$
\tau_{s_{m_{k}}} S_{a a}+\tau_{s_{m_{k}}} S_{c o r}=\tau_{s_{m_{k}}}\left(L_{\omega} T_{a a}\right)+\tau_{s_{m_{k}}}\left(L_{\omega} T_{c o r}\right)=\left(L_{\omega, k} \tau_{s_{m_{k}}} T_{a a}\right)+\left(L_{\omega, k} \tau_{s_{m_{k}}} T_{c o r}\right),
$$

By (5.5), (5.6), (5.7) and Lemma 1, the limits

$$
\lim _{k \rightarrow+\infty}\left(\tau_{s_{m_{k}}} S_{a a}+\tau_{s_{m_{k}}} S_{c o r}\right)=\lim _{k \rightarrow+\infty}\left(L_{\omega, k} \tau_{s_{m_{k}}} T_{a a}\right)+\lim _{k \rightarrow+\infty}\left(L_{\omega, k} \tau_{s_{m_{k}}} T_{c o r}\right),
$$

give

$$
\mathcal{P}=\tilde{L}_{\omega} \mathcal{V} \quad \text { on } \quad \mathbb{R}
$$

where

$$
\tilde{L}_{\omega}=\sum_{i=0}^{p} \sum_{j=0}^{q} \tilde{a}_{i j} \frac{d^{i}}{d x^{i}} \tau_{\omega_{j}}+K * .
$$

Consequently by (5.6) we obtain

$$
\lim _{k \rightarrow+\infty} \tau_{-s_{m_{k}}} \mathcal{P}=\lim _{k \rightarrow+\infty}\left(\tilde{L}_{\omega, k} \tau_{-s_{m_{k}}} \mathcal{V}\right) \quad \text { on } \quad \mathbb{R}
$$

where

$$
\tilde{L}_{\omega, k}=\sum_{i=0}^{p} \sum_{j=0}^{q} \tau_{-s_{m_{k}}} \tilde{a}_{i j} \frac{d^{i}}{d x^{i}} \tau_{\omega_{j}}+K *,
$$

which gives

$$
S_{a a}=L_{\omega} T_{a a} \quad \text { on } \quad \mathbb{R} .
$$

Finally, the equation $S_{a a}+S_{c o r}=L_{\omega} T_{a a}+L_{\omega} T_{\text {cor }}$ on $\mathbb{J}$ implies

$$
S_{\text {cor }}=L_{\omega} T_{\text {cor }} \quad \text { on } \quad \mathbb{J},
$$


hence the conclusion is true.

Remark 8. The proof of the theorem appeals to Lemma 1 and particulary to Proposition 9 characterisating the introduced space of smooth asymptotically almost automorphic functions.

Remark 9. The result of the theorem remains valid if we consider systems. Other problems can be tackled within the space of asymptotically almost automorphic distributions.

The following result concerns primitives.

Corollary 3. Let $S \in \mathcal{B}_{\text {aaa }}^{\prime}$, the following propositions are equivalent:

(1) $T \in \mathcal{B}_{\text {aaa }}^{\prime}$ is a primitive of $S$ on $\mathbb{J}$.

(2) There exist $V \in \mathcal{B}_{a a}^{\prime}$ a primitive on $\mathbb{R}$ of $S_{a a}$ and $W \in \mathcal{B}_{+, 0}^{\prime}$ a primitive of $S_{\text {cor }}$ on $\mathbb{J}$ such that

$$
T=V+W \quad \text { on } \quad \mathbb{J} \text {. }
$$

\section{REFERENCES}

1. Bochner S. Uniform convergence of monotone sequences of functions. Proc. Natl. Acad. Sci. USA, 1961. Vol. 47, No. 4. P. 582-585. DOI: 10.1073/pnas.47.4.582

2. Bochner S. A new approach to almost periodicity. Proc. Natl. Acad. Sci. USA, 1962. Vol. 48, No. 12. P. 2039-2043. DOI: $10.1073 /$ pnas.48.12.2039

3. Bochner S. Continuous mappings of almost automorphic and almost periodic functions. Proc. Natl. Acad. Sci. USA, 1964. Vol. 52, No. 4. P. 907-910. DOI: 10.1073/pnas.52.4.907

4. Bohr H. Almost Periodic Functions. Chelsea Publishing Company, 1947. 113 p.

5. Bouzar C., Khalladi M. T. Tchouar F. Z. Almost automorphic generalized functions. Novi Sad J. Math., 2015. Vol. 45, No. 1. P. 207-214. DOI: 10.30755/NSJOM.GF14.01

6. Bouzar C., Tchouar F.Z. Almost automorphic distributions. Mediterr. J. Math., 2017. Vol. 14. Art. No. 151. DOI: 10.1007/s00009-017-0953-3

7. Cioranescu I. Asymptotically almost periodic distributions. Appl. Anal., 1990. Vol. 34, No. 3-4. P. 251259. DOI: /10.1080/00036818908839898

8. Levitan B. M., Zhikov V. V. Almost Periodic Functions and Differential Equations. Cambridge University Pres, 1982. 224 p.

9. Fréchet M. Les fonctions asymptotiquement presque périodiques. Revue Sci., 1941. Vol. 79. P. 341-354.

10. Kostić M. Asymptotically Almost Periodic and Asymptotically Almost Automorphic Vector Valued Generalized Functions. 2018. 18 p. arXiv:1808.03163v1 [math.FA]

11. Kostić M., Pilipović S., Velinov D. Quasi-asymptotically almost periodic vector-valued generalized functions. Sarajevo J. Math., 2019. Vol. 15(28), No. 2. P. 181-199.

12. N'Guérékata G. M. Some remarks on asymptotically almost automorphic functions. Riv. Math. Univ. Parma, 1987. Vol. 13. P. 301-303.

13. Schwartz L. Théorie des Distributions. Paris: Hermann, DL, 1966.

14. Seeley R. T. Extension of $\mathcal{C}^{\infty}$ functions defined in a half space. Proc. Amer. Math. Soc., 1964. Vol. 15, No. 4. P. 625-626. DOI: 10.1090/S0002-9939-1964-0165392-8

15. Sobolev S. L. Applications of Functional Analysis in Mathematical Physics. American Mathematical Society, 1963. 239 p.

16. Stepanov V.V. Sur Quelques Généralisations des Fonctions Presque Périodiques. Paris: C. R. Acad. Sci., 1925. Vol. 181, P. 90-92.

17. Tchouar F.Z. Asymptotically almost automorphic generalized functions. Abstr. of the Int. Conf. GF 2016, September 4-9, Dubrovnik, 2016. P. 67.

18. Zaki M. Almost automorphic solutions of certain abstract differential equations. Ann. Mat. Pura Appl. (4), 1974. Vol. 101. P. 91-114. 